\title{
El lugar de la fuerza ilocucionaria en las prácticas lingüísticas. Un abordaje del lenguaje desde las perspectivas de Habermas y Bourdieu
}

\author{
Carolina Modenutti \\ Universidad Nacional del Nordeste (Argentina) \\ dcmodenutti@gmail.com
}

Recibido: 08/07/2015

Aceptado: 15/08/2015

Resumen: En el presente trabajo analizamos las concepciones de lenguaje de Habermas y Bourdieu, autores que combinan la perspectiva pragmática del lenguaje con sus teorías del funcionamiento social. Utilizaremos como eje de nuestro análisis la continuación y ampliación que ambos autores realizan de la teoría austiniana de los actos de habla, más precisamente la distinción entre fuerza ilocucionaria y fuerza perlocucionaria. De este modo, pretendemos transitar las concepciones de lenguaje y prácticas lingüísticas de Habermas y Bourdieu, intentando precisar sus puntos de coincidencia y de disidencia con el fin de alcanzar dos objetivos. En primer lugar, analizar las condiciones que hacen posible el desarrollo del lenguaje en el marco de las prácticas sociales $y$, en segundo lugar, examinar el lugar que cada uno de los autores otorga a la fuerza ilocucionaria en las emisiones lingüísticas y las funciones del lenguaje que devienen de dicha interpretación.

Palabras claves: lenguaje, prácticas sociales, funciones del lenguaje, Habermas, Bourdieu. 
Abstact: In this paper we will analyze Habermas and Bourdieu's conceptions of language, authors who combine linguistic pragmatism perspective with their theories on social functioning. We will take as axis of our analysis the continuation and extension which those thinkers made of Austin's theory of speech acts, more precisely the distinction between illocutionary force and perlocutionary force. Thus, our idea is to go trough the conceptions of Habermas and Bourdieu upon language and linguistic practices, trying to highlight points of agreement and disagreement, following two goals. Firstly, the analysis of the conditions which made possible the development of language within the frame of social practices. And secondly, the examination of the place each author grants to the illocutionary force in the linguistic emissions and the language functions that emerge from such reading.

Key words: language, social practices, language functions, Habermas, Bourdieu

Las teorías del lenguaje de Habermas y Bourdieu representan una integración de los planteos iniciales del pragmatismo de Wittgenstein y Austin, como así también una ampliación de dichos planteos pragmáticos ya que sus líneas de análisis se insertan en el desarrollo de las teorías de la sociedad. Si bien tanto Habermas como Bourdieu, cada uno según sus intereses de base, retoman y discuten varios pensadores y líneas tanto de la filosofía del lenguaje como de la sociología, en el presente artículo nos focalizaremos en el planteo del análisis pragmático del lenguaje de ambos autores teniendo como eje la continuación y ampliación de la teoría austiniana de los actos de habla, más precisamente la distinción entre fuerza ilocucionaria y fuerza perlocucionaria. No se encuentra dentro de nuestros objetivos desarrollar la teoría de Austin, puesto que no pretendemos mostrar en detalle la discusión que Habermas y Bourdieu entablan con él, sino que nuestra intención está puesta en analizar la utilización que éstos hacen de la distinción de los actos ilocucionarios y perlocucionarios. 
De este modo, pretendemos transitar las concepciones de lenguaje y prácticas lingüísticas de Habermas y Bourdieu, intentando precisar sus puntos de coincidencia y de disidencia con el fin de alcanzar dos objetivos. En primer lugar, analizar las condiciones que hacen posible el desarrollo del lenguaje en el marco de las prácticas sociales y, en segundo lugar, examinar el lugar que cada uno de los autores otorga a la fuerza ilocucionaria en las emisiones lingüísticas y las funciones del lenguaje que devienen de dicha interpretación. En vistas de lo señalado, estructuramos el artículo en dos partes. En la primera parte, nos abocamos al análisis de los conceptos centrales de la teoría de la acción comunicativa habermasiana para lo cual trabajamos específicamente con los textos Teoría de la acción comunicativa I. Racionalidad de la acción y racionalización social y Teoría de la acción comunicativa II. Crítica de la razón funcionalista. En la segunda parte, desarrollaremos las nociones principales de la teoría bourdieuana del lenguaje, para la cual nos centramos principalmente en el texto ¿Qué significa hablar? Economía de los intercambios lingüísticos. 


\section{1) Lenguaje y acción comunicativa en Habermas}

Habermas denomina pragmática universal a la tarea de reconstrucción de las condiciones universales que permiten alcanzar el acuerdo mediante el lenguaje; dicha reconstrucción la sitúa en el análisis de la acción comunicativa puesto que es el único tipo de acción orientada al entendimiento, mientras que las demás formas de acción lingüística posible son derivadas de ésta.

Lo que corresponde a la pragmática universal es el estudio de las emisiones tanto en clave de una teoría de la gramática universal, interesada en la reconstrucción no de las reglas de una determinada lengua o de un hablante particular sino de las reglas que subyacen a la capacidad de proferir oraciones en cualquier situación, como de una teoría de los actos ilocucionarios centrada en el establecimiento de relaciones intersubjetivas orientadas a lograr el entendimiento. ${ }^{1}$

En este sentido, el análisis en torno del acto de habla comprende estos dos aspectos de la comunicación: la emisión de contenidos proposicionales acerca de algo y la fuerza ilocucionaria que permite entenderse con los demás.

Habermas contrapone el modelo de la acción comunicativa al modelo de acción racional con arreglo a fines; ${ }^{2}$ este último supone que el actor orienta su acción según una meta precisa, elige los medios adecuados para alcanzarla y considera las consecuencias previsibles de su acción. Los efectos de la acción comprenden los resultados de la acción en relación con el fin deseado, las consecuencias previstas por el actor, como así también,

\footnotetext{
${ }^{1}$ Alegre, Javier. Concepciones institucionalistas del lenguaje en la perspectiva pragmática contemporánea. La Plata, Tesis Doctoral, 2011 (inédita), p. 203.

2 Habermas toma el modelo de acción racional con arreglo a fines de la tipología de la acción de Weber, la cual establece cuatro tipos de acción: racional con arreglo a valores, racional con arreglo a fines, afectiva y tradicional. Sin embargo, Habermas critica la tipología de Weber puesto que a su entender el criterio de clasificación de las acciones pareciera ser los diferentes grados de racionalización, lo cual implicaría considerar las acciones a partir de la relación medio-fin (concebida monológicamente) y no como relaciones sociales. Es por esto que para Habermas el planteo weberiano es una estructura conceptual estrecha donde sólo cabe juzgar a las acciones sociales bajo el aspecto de la racionalidad con arreglo a fines. Para considerar los aspectos de la acción susceptibles de racionalización que Weber pasa por alto, Habermas propone una clasificación de las acciones sociales según dos orientaciones básicas que corresponden a la coordinación de la acción por tramas de intereses y a la coordinación por acuerdo normativo. Cfr. Teoría de la acción comunicativa I. Racionalidad de la acción y racionalización social. Madrid, Taurus, 2003, pp. 359-367.
} 
las consecuencias no previstas. En consecuencia, Habermas distingue entre acciones orientadas al éxito y acciones comunicativas. El primer grupo lo constituyen, por un lado, las acciones instrumentales que son consideradas bajo el aspecto de observancia de reglas de acción técnicas y son evaluada por el grado de eficacia de la intervención y, por otro lado, las acciones estratégicas que son consideradas bajo el aspecto de observancia de reglas de elección racional y se evalúan por la influencia ejercida sobre las decisiones de un oponente racional. En cambio, las acciones comunicativas se coordinan por actos de entendimiento y los actores persiguen sus fines individuales pero de manera que su acción pueda armonizarse sobre la base de una definición compartida de la situación. ${ }^{3}$ De este modo, para Habermas las acciones sociales concretas pueden distinguirse según los participantes se orienten estratégicamente o comunicativamente. Las acciones estratégicas son acciones en las cuales la actitud de los agentes se encuentra orientada al éxito y para la consecución de su fin se basan en cálculos egocéntricos que persiguen intereses individuales. En este tipo de acción los participantes no se encuentran en igualdad de condiciones y existe la posibilidad que los agentes utilicen la manipulación o violencia en pos de las pretensiones de poder. Las acciones comunicativas por su parte se caracterizan por estar coordinadas mediante actos orientados entendimiento.

En la comunicación las interacciones de los sujetos se articulan no sólo en referencia a las cosas del mundo objetivo, sino también a la normatividad existente (mundo social) y a las propias vivencias internas (mundo subjetivo). La estructura de las interacciones que remiten a cualquiera de estos mundos está dada por la coordinación de las acciones mediante actos comunicativos, es decir, por acciones que a través de la utilización del lenguaje tienden a la obtención de acuerdos; desde esta perspectiva, la estructura social puede concebirse como producto de acciones entre sujetos que se entienden acerca del mundo con el fin de alcanzar acuerdos y coordinar las acciones para ello. En este tipo de acciones comunicativas la motivación se encuentra en la racionalidad

\footnotetext{
${ }^{3}$ Cfr. Ibíd., pp. 366-7.
} 
de los procesos de entendimiento y no en la manipulación de los actores o coacciones externas como en las acciones estratégicas, ya que para Habermas

un acuerdo alcanzado comunicativamente tiene que tener una base racional; es decir, no puede venir impuesto por ninguna de las partes, ya sea instrumentalmente, merced a una intervención directa en la situación de acción, ya sea estratégicamente, por medio de un influjo calculado sobre las decisiones de un oponente... El acuerdo se basa en convicciones comunes. ${ }^{4}$

La posibilidad de esta afirmación se deriva del hecho de que el entendimiento es la función paradigmática del lenguaje, en tanto las demás funciones que pueden darse en acciones mediadas lingüísticamente son derivadas o secundarias. Es decir, si bien no siempre las acciones mediadas lingüísticamente tienen por finalidad el entendimiento, como en los casos de entendimiento indirecto o en los casos de instrumentalización de la práctica comunicativa para el éxito de una acción individual, sigue siendo el entendimiento como proceso de obtención de acuerdo la función paradigmática. En palabras de Habermas, “...el lenguaje orientado al entendimiento, es el modo original, frente al entendimiento indirecto, la comprensión indirecta, el dar a entender o el hacer que el otro conciba tal o cual opinión, se comportan de forma parásita". 5 Para fundamentar su posición introduce en este punto la distinción de Austin entre fuerzas ilocucionarias y perlocucionarias. $^{6}$

\footnotetext{
${ }^{4}$ Ibíd., pp. 368-9.

${ }^{5}$ Ibíd., p. 370.

${ }^{6}$ En la tarea de mostrar las diferencias que existen entre las expresiones lingüísticas realizativas y las constativas, Austin propone considerar tres dimensiones o tipos de actos que pueden darse en un acto de habla. En primer lugar, el acto de decir algo constituye un acto locucionario, en el cual Austin incluye el acto fonético (emisión de ruidos), el acto "fático" (emisión de ciertos términos o palabras) y el acto "retico" (utilización de los términos con un cierto sentido y referencia, más o menos definidos). En segundo lugar, el acto ilocucionario es el acto que se realiza al decir algo (ordenar, afirmar, incitar, etc.). Y en tercer lugar, el acto perlocucionario es el acto que al decir algo produce consecuencias o efectos sobres los sentimientos, pensamientos o acciones del auditorio, o de quién emite la expresión, o de otras personas. [Cfr. Austin, John. Cómo hacer cosas con palabras. Barcelona, Paidós, 1990]. Esta distinción se inserta en los análisis austinianos respecto del uso y el carácter convencional del lenguaje y es retomada por Habermas y Bourdieu que se esfuerzan en precisar el lugar de la fuerza ilocucionaria en las prácticas comunicativas.
} 
Según Habermas, en los actos ilocucionarios el emisor se expresa para ser entendido y aceptado y en el significado manifiesto de la emisión quedan expresados la intención comunicativa y el objetivo ilocucionario. En cambio, los actos perlocucionarios no se derivan completamente del contenido manifiesto de la emisión sino que dependen de factores externos y contingentes; en este sentido

...Austin concluyó que los éxitos ilocucionarios guardan con el acto de habla una relación interna o regulada por convención, mientras los efectos perlocucionarios permanecen externos a lo dicho. Los posibles efectos perlocucionarios de un acto de habla dependen de contextos contingentes y, a diferencia de los éxitos ilocucionarios, no vienen fijados por convenciones. ${ }^{7}$

Si evaluamos las acciones sociales concretas en relación con los conceptos austinianos de fuerzas ilocucionarias y perlocucionarias, resulta claro que en la acción comunicativa los hablantes sólo persiguen con sus actos de habla fines ilocucionarios; el acuerdo se alcanza cuando el oyente capta el significado de la emisión, reconoce (o no) la pretensión de validez vinculada al acto de habla y orienta su acción según las convenciones establecidas. En cambio, en la acción estratégica los actos de habla se orientan a la consecución del éxito y en este plano surgen los efectos perlocucionarios. El significado explícito de la emisión pasa a tener una relación contingente con los efectos perlocucionarios ya que sólo puede conocerse el objetivo perlocucionario esclareciendo la intención del hablante, la cual no se deduce de lo expresado. Los actos perlocucionarios constituyen acciones estratégicas en la medida en que para lograr el efecto perlocucionario buscado el hablante debe ocultar o disfrazar su intención, generando así condiciones asimétricas en la interacción. No obstante, Habermas destaca que un acto de habla perlocucionario sólo puede tener éxito en tanto la emisión cumpla con los fines ilocucionarios, puesto que si no se logra el entendimiento no sería posible producir efectos perlocucionarios en el receptor, de manera que “...lo que al principio llamábamos "empleo del lenguaje orientado hacia las consecuencias" no es un modo originario del uso

\footnotetext{
${ }^{7}$ Ibíd., p. 374.
} 
del lenguaje, sino la subsunción de actos de habla que sirven a fines ilocucionarios, bajo las condiciones de la acción orientada al éxito". 8 Para que el hablante pueda actuar estratégicamente es necesario lograr su propósito ilocucionario y que el oyente entienda el significado de la emisión y se involucre en la aceptación de las pretensiones de validez, si bien esta oferta se presenta con ocultamientos y engaños. Es por ello que la función original del lenguaje es el entendimiento.

Una vez establecido el entendimiento como la función original del lenguaje, resulta necesario especificar sus condiciones de posibilidad, esto es, competencias comunicativas y pretensiones de validez presentes en los actos de habla para alcanzar el acuerdo. En cuanto a las competencias comunicativas, Habermas plantea que el entendimiento sólo puede darse en aquellos agentes que sean capaces de comportamientos o emisiones de los que puedan dar cuenta mediante buenas razones frente a un enjuiciamiento objetivo, es decir, que sus acciones sean susceptibles de fundamentación y de crítica por parte de quienes participan en la interacción.

Este concepto de racionalidad comunicativa posee connotaciones que, en última instancia, se remontan a la experiencia central de la capacidad de aunar sin coacciones y generar consenso que tiene un habla argumentativa, en que diversos participantes superan la subjetividad inicial de sus respectivos puntos de vista y merced a una comunidad de convicciones racionalmente motivada se aseguran, a la vez, de la unidad del mundo objetivo y de la intersubjetividad del contexto en que desarrollan sus vidas. ${ }^{9}$ Las acciones comunicativas pueden realizarse a partir de una capacidad subjetiva de generar consenso sin coacciones mediante un habla argumentativa en base a una serie de convicciones cuya motivación es racional (no violenta ni inducida).

Por otra parte, el acuerdo buscado en la práctica comunicativa no es simplemente por referencia a algo en los mundos objetivo, subjetivo o normativo, sino además basadas

\footnotetext{
${ }^{8}$ Ibíd., p. 375.

${ }^{9}$ Ibíd., p. 27.
} 
en las condiciones de validez necesarias para la comunicación. Habermas propone ampliar el concepto de pretensiones de validez de manera de no reducirlo a la validez veritativa de las proposiciones anclada en el plano semántico, sino que incluya además el plano pragmático de las emisiones, ${ }^{10}$ que puede estar relacionado con cada una de las posibles relaciones entre actor y mundo: mundo objetivo, mundo social y mundo subjetivo. Así, en cuanto a pretensiones de validez podemos hablar de verdad cuando el contenido proposicional de la enunciación refleja una experiencia o hecho capaz de ser compartido y da a entender algo en relación al exterior, al mundo objetivo; de rectitud cuando el componente ilocucionario de la emisión debe adecuarse al contexto normativo existente y debe entenderse con los demás según las normas y valores vigentes y corresponde al mundo social; hablamos de veracidad cuando el componente lingüístico debe coincidir con lo que el hablante piensa de la situación y expresar sus propias intenciones, debe darse a entender y corresponde al mundo subjetivo, su función es expresar vivencias subjetivas.

En la acción comunicativa las manifestaciones se vinculan simultáneamente con el mundo subjetivo, el mundo social y el mundo objetivo (aunque explícitamente el contenido proposicional refiera a uno de estos mundos) y llevan implícitas las pretensiones de validez constitutivas de los tres mundos; si la validez de la emisión es susceptible de crítica por otro actor significa que hay un sistema compartido que permite el entendimiento y el acuerdo, debido a que "entendimiento (Verständgung) significa "la obtención de un acuerdo" (Einigung) entre los participantes en la comunicación acerca de la validez de una emisión; acuerdo (Einverständnis), el reconocimiento intersubjetivo de la pretensión de validez que el hablante vincula en ella" ${ }^{11}$ En este sentido, la fuerza ilocucionaria de una emisión está en estrecha relación con el reconocimiento de las pretensiones de validez en tanto es "el componente que especifica qué pretensión de

${ }^{10}$ Cfr. Ibíd., p. 357.

${ }^{11}$ Habermas, Jürgen. Teoría de la acción comunicativa Il. Crítica de la razón funcionalista. Madrid, Taurus, 2003, p.171. 
validez plantea el hablante con su emisión, cómo la plantea y en defensa de qué lo hace" ${ }^{12}$ En otras palabras, en las pretensiones de validez reside la fuerza ilocucionaria que es la dimensión de la acción en la que se da el entendimiento.

Para concluir este apartado mostramos cómo se articulan las nociones desarrolladas hasta aquí a fin de sintetizar la concepción habermasiana de lenguaje. La práctica comunicativa se configura en relación con la normatividad vigente, las autopresentaciones expresivas y las cosas existentes en el mundo objetivo y funciona a partir de un suelo común de convicciones; dicho suelo común se fundamenta en el reconocimiento intersubjetivo de las pretensiones de validez susceptibles de crítica y sin necesidad de coacciones. La racionalidad de la práctica se expresa en la motivación racional del acuerdo y la racionalidad del agente en su capacidad para fundamentar mediante el habla sus acciones cuando las circunstancias lo requieran. El lenguaje funciona como medio para alcanzar el consenso entre los interlocutores, y cualquier otra función del lenguaje que difiera del entendimiento es derivada o parasitaria de este modo original. Las pretensiones de validez representan la dimensión ilocucionaria, las cuales dependen de las condiciones universales del lenguaje y se efectivizan en el uso que realiza cada hablante.

\section{2) Bourdieu: el lenguaje como medio de dominación}

Para comprender la práctica lingüística, y en función de ella al lenguaje, desde la perspectiva de Bourdieu es preciso situarnos en su concepción general de la acción. Para el autor, existe una determinación mutua y permanente entre la configuración de los esquemas de percepción, acción y discurso que forman los habitus y los campos donde estos habitus se constituyen, se desarrollan y se transforman. En lo que refiere al lenguaje, los agentes forman disposiciones lingüísticas en directa vinculación con su posición en el

\footnotetext{
${ }^{12}$ Habermas, Jürgen. Teoría de la acción comunicativa I... Op. Cit., p. 357.
} 
campo lingüístico y el estado de las luchas que allí dentro se juegan. Como resultado de estas condiciones cada agente es propenso a producir discursos referentes a ciertos temas, con características gramaticales semejantes y a utilizarlos en circunstancias determinadas. Asimismo, la disposición lingüística propende a expresarse mediante conductas y modales determinados, a la manera de una hexis corporal que explicita en el cuerpo las conminaciones del orden social. Así la utilización incorrecta de las palabras, el desconocimiento de su significado o un discurso inapropiado para la circunstancia, se manifiestan en nerviosismos, sonrojos, timidez, etc., y de igual forma, reconocer la correcta utilización de una lengua permite desenvoltura, fortaleza en la voz, firmeza en las palabras, etc. Para Bourdieu, el conocimiento que cada agente tiene del valor que portan sus discursos y que lo llevan a actuar en consecuencia proviene de la experiencia de su posición social:

...indudablemente la relación originaria con los diferentes mercados y la experiencia de las sanciones impartidas a las propias producciones constituyen, juntamente con la experiencia del precio concedido al propio cuerpo, una de las mediaciones a través de las cuales se constituye una especie de sentido personal del propio valor social que regula la relación práctica con los diferentes mercados (timidez, desenvoltura, etc.) y, más generalmente, toda la manera de comportarse en el mundo social. ${ }^{13}$

La competencia lingüística expresa, por un lado, la capacidad de articular discursos devenidos de la posición social y la experiencia de la misma y, por otro lado, el sentido práctico $^{14}$ de utilizar los discursos apropiadamente en relaciones con las posibilidades e

${ }^{13}$ Bourdieu, Pierre. ¿Qué significa hablar? Economía de los intercambios lingüísticos. Madrid, Akal, 1999, p. 56.

${ }^{14}$ La noción de sentido práctico es central en la teoría de la acción de Bourdieu y con ella desestima las teorías que suponen un agente consciente y calculador: "cada agente tiene un conocimiento práctico, corporal, de su posición en el espacio social... un sentido de su lugar (actual y potencial) convertido en un sentido de la colocación que rige su propia experiencia del lugar ocupado, definido absoluta, y sobre todo, relacionalmente, como puesto, y los comportamientos que ha de seguir para mantenerlo... y mantenerse en él". [Bourdieu, Pierre. Meditaciones pascalianas. Thomas Kauf, Barcelona, Anagrama, 1999, p. 242.] Sólo en situaciones extraordinarias el agente utiliza el cálculo racional, en la gran mayoría de las situaciones lo que guía las acciones es el sentido práctico, que las va adecuando en la práctica misma sin necesidad de reflexibilidad permanente y consciente, sino por la estructuración previa de las disposiciones y el 
imposibilidades que el campo permite y que en el agente se internaliza en forma de intereses. El interés que se expresa en el habla es una relación de creencia, de reconocimiento de valor y merecimiento de apuesta, que resulta de los presupuestos fundamentales del campo, de sus regularidades prácticas definidas a lo largo de la historia y de las variaciones estructurales. Esta noción de interés permite pensar que los agentes, en la regularidad de sus prácticas en general y de las prácticas lingüísticas en particular, no realizan cálculos conscientes especulando qué será lo más provechoso, qué deben decir, cómo articular sus discursos, etc., sino que más bien realizan un acto de fe, una relación de creencia, que sitúa en un plano pre-reflexivo los intereses del juego social. Este sentido práctico del funcionamiento del juego también permite conocer las potenciales sanciones o rechazos de quienes transgredan las condiciones sociales de la aceptabilidad;

las esperadas condiciones de recepción forman parte de las condiciones de producción y la anticipación de las sanciones del mercado contribuye a determinar la producción del discurso... Es esta forma de la aceptabilidad, y no ninguna forma de cálculo racional orientado hacia la maximización de los beneficios simbólicos, lo que, al incitar a que se tome en cuenta el valor probable del discurso en la producción, determina a su vez las correcciones y todas las otras formas de autocensura; concesiones que se conceden a un universo social por el hecho de hacerse aceptable para él. ${ }^{15}$

La denominación bourdieuana de mercado lingüístico para referirse al ámbito donde se desarrollan las prácticas lingüísticas se vincula con la lógica económica que lo rige; el capital lingüístico que portan (desigualmente) los agentes adquiere sentido y reconocimiento según el valor o precio fijado a las emisiones lingüísticas por el grupo dominante y no simplemente por los costos que insume su formación. La competencia lingüística se convierte en capital en tanto la lucha entre los participantes para lograr su

conocimiento práctico del sentido del juego. Las respuestas adaptadas al campo no pueden ser en todos los casos (en algunos casos sí) producto de un razonamiento previo debido a que la historia social y la trayectoria personal al momento de dar respuestas ponen en relación la multiplicidad de factores que constituyen al individuo como agente social, es decir que no se puede hacer abstracción de todas las determinaciones y relaciones de las que forma parte para actuar en un momento dado.

${ }^{15}$ Bourdieu, Pierre. ¿Qué significa hablar?...Op. cit., p.50-1. 
posesión es lo que da sentido a las apuestas, y además es lo que posiciona a los agentes en el campo y les permite distinguirse. Es condición necesaria para la dominación lingüística que un grupo reducido de agentes conserve el monopolio del capital dado que, en palabras de Bourdieu,

...el beneficio de distinción se debe de hecho a que la oferta de producto (o de locutores) correspondiente a un determinado nivel de cualificación lingüística (o cultural) es inferior a lo que sería si todos los locutores se beneficiarían de iguales condiciones de adquisición de la competencia legítima que la que disfrutan los poseedores de una competencia excepcional, ese beneficio se distribuye lógicamente en función de las posibilidades de acceso a estas condiciones, es decir, en función de la posición ocupada en la estructura social. ${ }^{16}$

De esto se deduce que, en primer lugar, las relaciones generadas dentro del mercado lingüístico a partir de la posesión de la competencia legítima está en gran parte determinada por condiciones exteriores al campo específico, esto es, con la distribución de otros capitales (económico, cultural, social, etc.) que influyen en la configuración global del espacio social; y en segundo lugar, la distribución del capital es muy desigual en función de los mecanismos de distribución que funcionan en la estructura social global y que se repiten en el campo lingüístico, y esto es lo que permite la existencia del beneficio de los dominantes. Veamos cómo funcionan estos mecanismos de distribución y qué implicancias tiene para el mercado lingüístico.

En la configuración específica del campo (su estructura), las significaciones y distinciones constituyen el orden simbólico que forma parte de la realidad del campo e interviene en el funcionamiento del orden material, dado que las atribuciones, características, limitaciones, prohibiciones, etc. otorgadas a una posición marcan un modo de ser y actuar que se ve reflejado en la realidad. Lo simbólico es un poder productor de realidades que legitima el orden social y desencadena la lucha por poseer los instrumentos necesarios para imponer, legitimar y reproducir una concepción de mundo.

\footnotetext{
${ }^{16}$ Ibíd., p. 30.
} 
Las fuerzas dominantes que buscan la reproducción ejercen dominación mediante la utilización de violencia simbólica, esto es, estrategias sutiles y efectivas que permiten la imposición de criterios y valores que representan sus intereses, cuyo efecto se ejerce en las disposiciones del habitus. La violencia simbólica actúa imponiendo una arbitrariedad cultural, conjunto de conocimientos, modos de vida, costumbres, lengua, etc., unificada y totalizada para un conjunto social, si bien no en forma de contenidos puntuales de ideas, principios, normas, sino como modalidades de sentido que se presentan como verdaderas, referidas por ejemplo a la existencia de una jerarquía cultural, la necesidad de preeminencia de un punto de vista, un criterio de autoridad oficializado, etc. ${ }^{17}$ Este mecanismo, por un lado, estructura las disposiciones subjetivas que dan forma a la percepción y evaluación del mundo mediante disciplinas mentales y corporales, y por otro clasifica, divide, incluye y excluye agentes, prácticas y discursos al establecer un criterio o principio como el legítimo.

En el caso del lenguaje, las distribuciones, posiciones, competencias y luchas del campo lingüístico se definen por referencia directa a la lengua oficial, esto es, a la lengua que se encuentra legitimada y reconocida por todos los participantes de la comunicación como dominante. Para que la lengua oficial perdure en el tiempo como tal y ciertos agentes puedan distinguirse por su excelencia lingüística es necesario que las instituciones y mecanismos de inculcación, formación y corrección realicen un trabajo permanente de distinción y corrección, de manera de especificar la lengua legítima y su manejo y de reforzar las diferencias entre los poseedores y los no poseedores de la misma. Para que esto sea posible, estos mecanismos de transmisión de la lengua (cultura, sistema educativo, familia, clase social, etc.) están dirigidos a que el conocimiento de la lengua

\footnotetext{
${ }^{17}$ En este sentido, afirma Bourdieu que para que la dominación pueda ser efectiva debe transcender el plano de la acumulación de bienes simbólicos y ocuparse de los mecanismos de distribución de los mismos: "la historia de los regímenes, las instituciones, los acontecimientos o las ideas no funcionan como cultura histórica, simple acumulación de bienes simbólicos cuyo fin se encuentra en sí misma, sino como método de percepción y de acción políticas, conjunto de esquemas operatorios que permiten engendrar, fuera de toda referencia, las situaciones originarias, discursos o acciones cargados con toda una experiencia histórica" [Bourdieu, P. y Boltanski, L. La producción de la ideología dominante. Trad. Heber Cardoso. Nueva Visión, Bs. As., 2009, p. 92]
} 
oficial sea altamente restringido; no obstante es imprescindible que el reconocimiento de la misma esté lo más extendido posible, inscrito en los habitus, y a su vez, sustentado en un desconocimiento de la violencia que ejerce sobre los agentes. Esta configuración de conocimiento, reconocimiento y desconocimiento que posibilita la dominación simbólica, es a la vez lo que posibilita las luchas y cambios lingüísticos, "en efecto, las luchas lingüísticas que son origen de estos cambios suponen locutores que tengan (más o menos) el mismo reconocimiento del uso autorizado y de los conocimientos desiguales de este uso". 18

Bourdieu critica el modelo de la filosofía intelectualista que entiende el lenguaje de manera esencialista y lo toma como objeto de intelección. Desde esta perspectiva, el mundo social se configura simplemente como universo de intercambios simbólicos y la acción lingüística se reduce a su función comunicativa; ${ }^{19}$ para Bourdieu, por el contrario, el lenguaje es un instrumento de acción y de poder y no un mero medio de comunicación. A partir de lo expuesto respecto del mercado lingüístico resulta claro que a las acciones comunicativas subyacen relaciones de dominación y los agentes que intervienen en la comunicación se encuentran en relaciones de poder donde el lenguaje funciona como medio de dominación: "los intercambios lingüísticos son relaciones de comunicación pero también relaciones de poder simbólico donde se actualizan las relaciones de fuerza entre los locutores y sus respectivos grupos". ${ }^{20}$ De esta manera, el lenguaje no es sólo el portador de una lengua, sino de discursos que guardan un estilo particular y funcionan como signo de distinción, definen la experiencia del agente con los discursos que emite a nivel corporal, a nivel cognitivo y a nivel social-político en tanto dicha emisión lo posiciona en el campo lingüístico: "el lenguaje es una técnica corporal y la competencia

\footnotetext{
${ }^{18}$ Bourdieu, Pierre. ¿Qué significa hablar?... Op. cit., p.36.

${ }^{19} \mathrm{Cfr} .:$ "Si el modelo lingüístico se ha transportado tan fácilmente al terreno de la etnología y de la sociología, ello se ha debido a una consideración esencialista de la lingüística, es decir, a la filosofía intelectualista que hace del lenguaje, más que un instrumente de acción y de poder, un objeto de intelección. Aceptar el modelo saussuriano y sus presupuestos, es tratar el mundo social como universo de intercambios simbólicos y reducir la acción a un acto de comunicación que, como la palabra de Saussure, está destinado a ser descifrado por medio de una cifra o de un código, lengua o cultura". Ibíd., p. 11.

${ }^{20}$ Ibíd., p. 11
} 
propiamente lingüística, y muy especialmente fonológica, es una dimensión de la hexis corporal donde se expresan toda la relación del mundo social y toda la relación socialmente instruida con el mundo". ${ }^{21}$ No existe un lenguaje neutro, las connotaciones sociales condicionan en gran medida el habla y la actitud de los agentes en tanto otorgan significado y eficacia a los actos de habla.

Los factores que intervienen para que una lengua se instituya como dominante trascienden no sólo el ámbito formal de la semántica y gramática del lenguaje, sino además el ámbito propio del campo lingüístico,

...todas las prácticas lingüísticas se valoran con respecto a un patrón de las prácticas legítimas, las prácticas de los dominantes. Por eso, el valor probable que objetivamente corresponda a las producciones lingüísticas de los diferentes locutores, así como la relación que cada uno de ellos puede mantener con la lengua- y por tanto con su propia producción-, se define desde dentro del sistema de variables prácticamente competitivas que se instituye cada vez que existe las convicciones extralingüísticas de constitución de un mercado lingüístico. ${ }^{22}$

Las contingencias históricas de una sociedad, factores económicos, políticos, culturales, definen de manera externa al campo lingüístico las posiciones dominantes que cuentan con las prerrogativas de imponer la lengua hegemónica y oficial. De esta manera, el ejercicio de la violencia simbólica para asegurar el poderío del grupo dominante se expresa en la asignación de nominaciones, atribuciones, clasificaciones, jerarquizaciones, etc. que implican las exigencias de comportamientos, de límites, de maneras de ser, que forman los habitus y se expresan en hexis corporales:

Instituir, asignar una esencia, una competencia, es imponer un derecho de ser que es un deber ser (o un deber de ser). Es significar a alguien lo que es y significarle que tiene que conducirse consecuentemente a como se le ha significado. El indicativo es en este caso un

\footnotetext{
${ }^{21}$ Ibíd., pp. 59-60.

22 Ibíd., p. 27.
} 
imperativo. Instituir, dar una definición social, una identidad, es también imponer límites. Así el acto de institución es un acto de comunicación: significa a alguien su identidad. ${ }^{23}$

Ahora bien, para que las palabras logren instituir, es decir, logren tener un efecto en aquellos que las reciben, deben provenir de una voz autorizada que cuente con un reconocimiento social suficiente que permita que sus palabras causen los efectos deseados. El agente en la pronunciación de su discurso se sustenta no sólo en su competencia lingüística sino además en las instituciones que lo avalan y el estado de las relaciones de fuerzas que definen la situación actual del campo, de manera que el hablante representa una autoridad por encarnar en su discurso estos tres aspectos del lenguaje (competencia, institución, relaciones de fuerza).

La eficacia del discurso performativo que pretende el advenimiento de lo que enuncia en el acto mismo de enunciar es proporcional a la autoridad de quien lo enuncia. El mundo social es también representación y voluntad y existir socialmente, es también ser percibido, y percibido como diferente. $^{24}$

Las relaciones sociales institucionalizadas y el estado de las relaciones de fuerza dentro del mercado lingüístico permiten a los dominantes que sus producciones funcionen como criterios de visión, apreciación y clasificación, es decir, construir visiones de mundo que claramente contribuyen a favorecer sus posiciones y asegurar la reproducción de las condiciones que permiten su dominación. Con esto Bourdieu demuestra que en los discursos, la competencia técnica y la lógica propiamente lingüística del discurso van acompañadas por un conjunto de condiciones sociales y de relaciones de dominación que la estructuran. "La eficacia mágica de esos actos de institución es inseparable de la existencia de una institución que defina las condiciones (en materia de

\footnotetext{
${ }^{23}$ Ibíd., p.81.

${ }^{24}$ Ibíd., p.91.
} 
agente, de lugar o de momento, etc.) que deban reunirse para que la magia de las palabras puedan actuar". 25

En el plano de las interacciones lingüísticas, la eficacia del habla no depende de las ilocuciones, ni del sistema simbólico del lenguaje sino del contexto institucional que rodea al hablante y a su discurso; la capacidad realizativa del lenguaje se sustenta en las condiciones sociales y no sólo a partir de las emisiones lingüísticas. La fuerza ilocucionaria de las expresiones no se encuentra en las palabras, sino en las condiciones sociales de producción de las mismas:

Tal es el principio de error de Austin y Habermas cuando creen descubrir en el propio discurso, es decir, en la sustancia propiamente lingüística de la palabra, su principio de eficacia. Intentar comprender lingüísticamente el poder de las manifestaciones lingüísticas, buscar en el lenguaje el principio de la lógica y la eficacia del lenguaje de institución, equivale a olvidar que la autoridad del lenguaje llega desde fuera. ${ }^{26}$

De igual manera, la performatividad del acto depende de la autoridad del emisor, motivo por el cual para Bourdieu nunca se dan condiciones de igualdad en el habla. Quien cuenta con suficiente capital lingüístico para convertirse en portavoz oficial y logra causar los efectos buscados con sus actos de habla necesariamente ha de formar parte del grupo de agentes dominantes dentro del campo lingüístico, pues su autoridad no se constituye solo por los efectos alcanzados sino porque la condición necesaria para ello es contar con el poderío de los medios de distribución del capital lingüístico (y los demás capitales en relación con éste) que aseguran la fuerza de sus palabras y la conservación de su posición dominante. En la posición opuesta, los agentes dominados se encuentran predispuestos a recibir y aceptar las condiciones de dominación implícitas en la comunicación.

\footnotetext{
${ }^{25}$ Ibíd., p.46.

${ }^{26}$ Ibíd., p.67.
} 


\section{Conclusión}

Llegados a este punto, retomamos los objetivos plateados en la introducción donde nos proponíamos, de manera más general, analizar en los planteos tanto de Habermas como de Bourdieu las condiciones que hacen posible el desarrollo del lenguaje en el marco de las prácticas sociales y, de manera más específica, examinar el lugar que cada uno de los autores otorga a la fuerza ilocucionaria en las emisiones lingüísticas y las funciones del lenguaje que devienen de dicha interpretación.

Para Habermas los sujetos capaces de acción y lenguaje entablan interacciones lingüísticas sobre la base de las pretensiones de validez de verdad, rectitud y veracidad que corresponden al mundo objetivo, social y subjetivo respetivamente. En tanto dichas pretensiones de validez puedan ser objeto de crítica por los receptores y fundamentadas por los emisores y puedan argumentarse con buenas razones sin que exista la necesidad de recurrir a formas inducidas o violentas de aceptación, las prácticas lingüísticas se mueven en un plano de racionalidad comunicativa que es inherente al lenguaje. De esta manera, la fuerza ilocucionaria de las emisiones se encuentra en la racionalidad de las mismas y no en condiciones exteriores al lenguaje; en consecuencia, el poder realizativo del lenguaje pertenece a su estructura interna. Esto permite afirmar a Habermas que la función primordial del lenguaje es el entendimiento, en tanto es la condición de posibilidad de cualquier otro tipo de función que se pretenda con el uso del lenguaje.

En Bourdieu encontramos una posición muy diferente. La producción de discursos en primera instancia surge de una capacidad natural de producción fonológica que es compartida universalmente, pero lo que determina las variaciones de discursos y la distinción entre agentes es la competencia social que refleja en el discurso las posesiones, posición y posibilidades de un agente dentro del campo lingüístico. El poder de causar efectos del lenguaje no deriva de sus propiedades intrínsecas sino de las condiciones externas que rodean la emisión de un discurso. Para Bourdieu, entonces, la fuerza ilocucionaria del lenguaje guarda una dependencia directa de las instituciones y relaciones 
de fuerza que determinan el escenario donde se origina y donde se recibe el discurso. De esta manera, es ingenuo pensar que el lenguaje puede expresar una función meramente comunicativa con vistas a alcanzar acuerdos. El lenguaje funciona como mecanismo que crea e instituye realidades y frente a esto el consenso no es su función primordial ni la más extendida, sino que por el contrario busca que la visión de mundo impuesta logre conservar y reproducir las condiciones de desigualdad que permiten la hegemonía de los medios de producción de discursos por parte de un grupo social determinado. En otras palabras, la función primordial del lenguaje no es la comunicativa, tal como lo concibe Habermas, sino la distinción de autoridad, estilo y los beneficios materiales y simbólicos que detenta la utilización de los discursos. No se busca la comprensión o el entendimiento sino la distinción y la dominación simbólica, dos notas que para la perspectiva bourdieuana son constitutivas de las relaciones sociales, en toda la extensión del espacio social, y de las relaciones lingüísticas dentro de su microcosmos específico particular.

Para concluir, podemos afirmar que tanto Habermas como Bourdieu rescatan el componente realizativo del lenguaje, que adquiere preeminencia con el planteo austiniano, pero a partir de condiciones diferentes. Para Habermas se encuentra en la fuerza ilocucionaria que es inmanente a la estructura del lenguaje, en tanto que para Bourdieu se encuentra más allá de la fuerza ilocucionaria, en las instituciones y relaciones de dominación del campo. Según nuestro parecer, la propuesta de Bourdieu tiene en cuenta en el análisis una serie de elementos que constituyen el funcionamiento global de las prácticas sociales, no solamente lingüísticas, lo cual amplía el espectro de análisis y brinda una concepción más amplia. Su interés por las condiciones sociales de producción, circulación y efectos de las prácticas lingüísticas lleva a sostener que si bien la capacidad realizativa del lenguaje se encuentra en parte en su estructura interna, dicha capacidad depende además de las condiciones que proporciona el campo lingüístico y los factores externos a dicho campo, propios de la dinámica de la sociedad y que también afectan su desarrollo. De manera que podemos afirmar que no hay una función original del lenguaje que deba cumplirse necesariamente en la comunicación y que en última instancia la 
función más extendida es la distinción y la dominación. Los agentes al actuar son más razonables que racionales en tanto sus prácticas se originan en habitus que tienen interiorizados estas formas de dominación y de subordinación. No hay racionalidad para llegar al consenso, sino razonabilidad para actuar coherente y según la lógica establecida en la dinámica social.

\section{Bibliografía}

- $\quad$ Alegre, Javier. Concepciones institucionalistas del lenguaje en la perspectiva pragmática contemporánea. La Plata, Tesis Doctoral, 2011 (inédita), pp. $184-210$ y $249-278$.

- $\quad$ Austin, John. Cómo hacer cosas con palabras. Barcelona, Paidós, 1990.

- Bourdieu, Pierre. ¿Qué significa hablar? Economía de los intercambios lingüisticos. Madrid, Akal, 1999.

- Bourdieu, Pierre. "Sobre el poder simbólico", en: Intelectuales, política y poder. Bs. As., Eudeba, 2005, pp. 65-73.

- Bourdieu, Pierre. El sentido práctico. Bs. As., Siglo XXI, 2010.

- $\quad$ Bourdieu, P. y Boltanski, L. La producción de la ideología dominante. Nueva Visión Bs. As., 2009.

- Bourdieu, Pierre. El sentido práctico. Siglo XXI, Bs. As., 2007.

- Bourdieu, Pierre. Meditaciones pascalianas. Barcelona, Anagrama, 1999.

- Habermas, Jürgen. Teoría de la acción comunicativa I. Racionalidad de la acción y racionalización social. Madrid, Taurus, 2003.

- Habermas, Jürgen. Teoría de la acción comunicativa II. Crítica de la razón funcionalista. Madrid, Taurus, 2003. 